\title{
Topology Optimization for Minimizing the Resonant Response of Plates with Constrained Layer Damping Treatment
}

\author{
Zhanpeng Fang and Ling Zheng \\ State Key Laboratory of Mechanical Transmission, Chongqing University, Chongqing 400044, China \\ Correspondence should be addressed to Ling Zheng; zling@cqu.edu.cn
}

Received 26 November 2014; Revised 1 March 2015; Accepted 6 March 2015

Academic Editor: Miguel Neves

Copyright ( 2015 Z. Fang and L. Zheng. This is an open access article distributed under the Creative Commons Attribution License, which permits unrestricted use, distribution, and reproduction in any medium, provided the original work is properly cited.

\begin{abstract}
A topology optimization method is proposed to minimize the resonant response of plates with constrained layer damping (CLD) treatment under specified broadband harmonic excitations. The topology optimization problem is formulated and the square of displacement resonant response in frequency domain at the specified point is considered as the objective function. Two sensitivity analysis methods are investigated and discussed. The derivative of modal damp ratio is not considered in the conventional sensitivity analysis method. An improved sensitivity analysis method considering the derivative of modal damp ratio is developed to improve the computational accuracy of the sensitivity. The evolutionary structural optimization (ESO) method is used to search the optimal layout of CLD material on plates. Numerical examples and experimental results show that the optimal layout of CLD treatment on the plate from the proposed topology optimization using the conventional sensitivity analysis or the improved sensitivity analysis can reduce the displacement resonant response. However, the optimization method using the improved sensitivity analysis can produce a higher modal damping ratio than that using the conventional sensitivity analysis and develop a smaller displacement resonant response.
\end{abstract}

\section{Introduction}

The CLD treatment has been regarded as an effective way to suppress structural vibrations and sound radiation. Among first studies of CLD treatments, Kerwin [1] developed a simplified theory to calculate the loss factor of a plate with CLD treatments. DiTaranto and Blasingame [2] extended Kerwin's work by accounting for the extensional deformations in the viscoelastic layer and obtained loss factors for three and five layer beams as a function of frequency. Mead and Markus [3] derived sixth-order equation of motion for beams in terms of transverse displacements. Most of these early works dealt with full coverage passive constrained layer damping (PCLD) treatments that are evidently not practical in purpose. For instance, in the damping treatment applications to the automotive and airplane industries, the weight constraint may not allow full PCLD coverage, since the added weight induced by any vibration and noise control design modification has to be limited to a small amount.
Therefore, a partial-coverage treatment is a more attractive approach to fulfill the real PCLD treatment requirements. Nokes and Nelson [4] were among the earliest investigators to provide the solution to the problem of a partially covered sandwich beam. The modal strain energy method was used to calculate modal loss factors of the treated beam for symmetric boundary conditions. Lall et al. [5, 6] performed a more thorough analytical study for partially covered planar structures. An important finding from the analysis was that for suitably chosen parameters higher values of the modal damping factor may be obtained for a partially covered beam compared to that obtained for a fully covered one under the condition of the same added weight of PCLD treatments. This encourages many researchers to investigate the optimal layout of CLD treatment in thin plates and shells.

Kodiyalam and Molnar [7] improved the modal strain energy (MSE) method through introducing a new method to account for viscoelastic material property variations with frequency. Lumsdaine [8] determined the optimal shape of 
a constrained damping layer on an elastic beam by means of topology optimization. The optimization objective is to maximize the system loss factor for the first mode of the beam. Zheng et al. [9] studied the optimal placement of rectangular damping patches for minimizing the structural displacement of cylindrical shells with the Genetic Algorithm. Moreira and Rodrigues [10] used MSE method to optimally locate passive constrained viscoelastic damping layers on structures. They also verified their work by comparing their results with experimental tests. Li and Liang [11] utilized the response surface method (RSM) to analyze and optimize the vibroacoustic properties of the damping structure. Ling et al. [12] studied the topology optimization for the layout of CLD in plates to suppress their vibration and sound radiation. The objective is to minimize structural modal damping ratios using the method of moving asymptotes (MMA). Lepoittevin and Kress [13] proposed a new method for enhancement of damping capabilities of segmented constrained layer damping material and developed an optimization algorithm using mathematical programming to identify a cuts arrangement that optimized the loss factor. Ansari et al. [14] studied the optimal number and location of the CLD sheets on the flat structure surface utilizing an improved gradient method and the objective was to maximize loss factor of the system. Kim et al. [15] compared the modal loss factors obtained by topology optimization to the conventional strain energy distribution (SED method) and the mode shape (MSO approach). It is found that topology optimization based on the rational approximation for material properties (RAMP) model and optimality criteria (OC) method can provide about up to 61.14 percent higher modal loss factor than SED and MSO methods. The numerical model and the topology optimization approach are also experimentally validated.

More recent attempts on the simultaneous shape and topology optimization of dynamic response have achieved some success. Rong et al. [16] developed the evolutionary structural optimization (ESO) method for the topology optimization of continuum structure under random dynamic response constraints. Zheng et al. [17] studied the optimal placement of rectangular damping patches for minimizing the structural displacement of cylindrical shells with the Genetic Algorithm. Pan and Wang [18] applied adaptive genetic algorithm to optimize truss structure with frequency domain excitations. The optimization constraints include displacement responses under force excitations and acceleration responses under foundation acceleration excitations. Alvelid [19] proposed a modified gradient of damping materials to mitigate noise and vibration. Yoon [20] proposed the topology optimization based on the SIMP method for the frequency response problem where the structure is subject to the wide excitation frequency domain. Shu et al. [21] propose level set based structural topology optimization for minimizing frequency response. The general objective function is formulated as the frequency response minimization at the specified points or surfaces with a predefined excitation frequency or a predefined frequency range under the volume constraint.

Although above optimal approaches can supply the design methods to reduce structural vibration and sound radiation in practice, the modal damping ratios for different modes are selected as the optimization objectives in these studies. In fact, the modal damping ratio is only the characteristics of vibration energy dissipation in structures rather than a controllable dynamic performance. Furthermore, the derivative of the modal damp ratio is usually neglected in conventional sensitivity analysis which will give rise to error in sensitivity analysis and produce a deviation from the optimal layout of CLD treatment in structures. This paper develops a dynamic topology optimization method of plates with CLD treatment under specified broadband harmonic excitation, which includes one or more resonance frequencies. The optimization objective is to minimize the square of displacement resonance response instead of conventional modal damping ratio. Meanwhile, an improved sensitivity analysis method with considering the derivative of modal damp ratio is developed in order to obtain the more accurate layout of CLD material on the plate and the lower displacement resonance response of CLD system.

\section{Statement of Optimization Formulation}

2.1. Dynamic Responses Using the Mode Superposition Method. The finite element equation for a structural dynamic problem under external harmonic excitations can be written in the form

$$
\mathbf{M} \ddot{X}+\mathbf{C} \dot{X}+\mathbf{K X}=f(t),
$$

where $\mathbf{M}, \mathbf{C}$, and $\mathbf{K}$ are the mass, damping, and stiffness matrices of the structure, respectively. $f(t)=F e^{i \omega_{f} t}$ is the vector of excitation force. $\omega_{f}$ and $F$ represent the frequency of the harmonic excitation and amplitude, respectively. $X$, $\dot{X}$, and $\ddot{X}$ denote the displacement, velocity, and acceleration vectors, respectively.

For a plate with CLD treatment, the matrices $\mathbf{M}$ and $\mathbf{K}$ can be further expressed as

$$
\begin{gathered}
\mathbf{M}=\mathbf{M}_{b}+\mathbf{M}_{v}+\mathbf{M}_{c}, \\
\mathbf{K}=\mathbf{K}_{b}+\mathbf{K}_{v}+\mathbf{K}_{c},
\end{gathered}
$$

where the subscript $b, v$, and $c$ denote the base plate, the viscoelastic damping layer, and the constrained layer, respectively.

For the CLD plate system, the $i$ th modal loss factor can be written by using MSE method [22]:

$$
\eta_{i}=\frac{\eta_{v} E_{s v}^{i}}{E_{s}^{i}}=\eta_{v} \frac{\sum_{j=1}^{n} E_{s v}^{i j}}{\sum_{j=1}^{n}\left(E_{s p}^{i j}+E_{s v}^{i j}+E_{s c}^{i j}\right)},
$$

where $\eta_{v}$ is the loss factor of the damping material. $E_{s v}^{i}$ is the $i$ th mode strain energy of the damping material. $E_{s}^{i}$ is the total $i$ th mode strain energy of CLD system. $E_{s p}^{i j}, E_{s v}^{i j}$, and $E_{s c}^{i j}$ are the $i$ th mode strain energy of the base material, damping layer, and constrained layer at jth element, respectively.

On the other hand, the relationship between modal loss factor and modal damping ratio is expressed as [23]

$$
\eta=2 \zeta \sqrt{1-\zeta^{2}}
$$




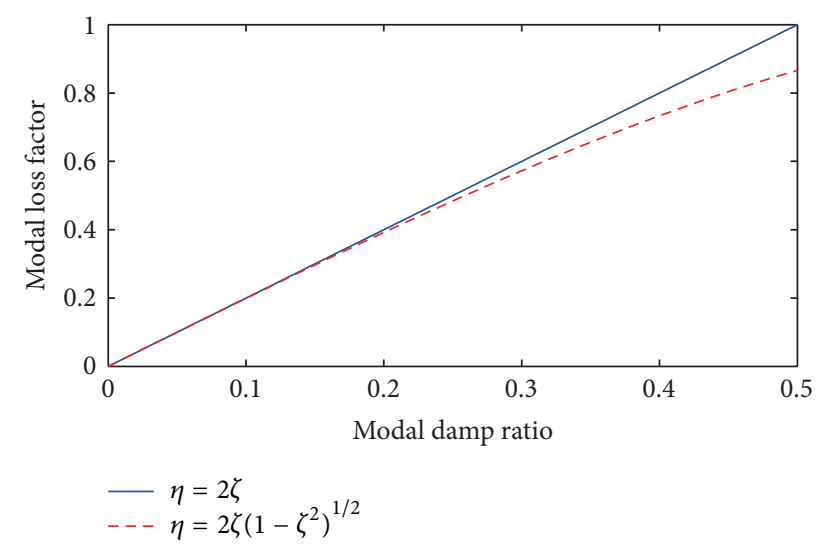

FIGURE 1: Relationship between loss factor $\eta$ and damping ratio $\xi$.

In fact, when modal loss factor is less than 0.3, the following expression can be used to calculate modal damping ratio instead of (4); the deviation is within $5 \%$. It can be seen from Figure 1. Consider

$$
\eta=2 \zeta
$$

Based on above analysis, it is assumed that the damping in CLD plate system can be expressed by using a proportional damping form. Equation (1) can be uncoupled as follows:

$$
\ddot{y}_{i}+2 \omega_{i} \zeta_{i} \dot{y}_{i}+\omega_{i}^{2} y_{i}=q_{i} \quad(i=1,2, \ldots, N),
$$

where $y_{i}$ is the modal coordinates defined by

$$
X=\left[\varphi_{1}, \varphi_{2}, \ldots, \varphi_{n}\right]\left\{\begin{array}{c}
y_{1} \\
y_{2} \\
\vdots \\
y_{n}
\end{array}\right\}=\boldsymbol{\Phi} y
$$

The displacement response in time domain is further expressed by using the mode superposition method:

$$
X\left(\omega_{f}\right)=\sum_{i=1}^{m} \frac{\varphi_{i} \varphi_{i}^{T} F}{\sqrt{\left(\omega_{i}^{2}-\omega_{f}^{2}\right)^{2}+\left(2 \zeta_{i} \omega_{f} \omega_{i}\right)^{2}}} .
$$

In the above equations, $\omega_{i}$ and $\varphi_{i}$ are the $i$ th circular frequency and mode shape normalized with respect to the mass matrix. $\zeta_{i}$ denotes the mode damping ratio of $i$ th mode. $q_{i}$ is the $i$ th element of $\Phi^{T} f(t)$ and is called the $i$ th generalized excitation. $m$ is the number of truncated modes.

When CLD plate system is subjected to harmonic excitations, the exciting frequency range is assigned to $\left[\omega_{l}, \omega_{t}\right]$, which includes one or several resonant frequencies. On the other hand, it is assumed that the system has a small damping and no repeated frequency in the range. Therefore, the displacement response at frequency $\omega_{i}$ is similar to single modal response, which can be expressed as follows:

$$
X\left(\omega_{i}\right)=\frac{\varphi_{i} \varphi_{i}^{T} F}{2 \zeta_{i} \omega_{i}^{2}} .
$$

2.2. Formulation of the Optimization Problem. In previous studies, the dynamic compliance is often considered as the objective function of dynamic optimization [24, 25]. It concentrates the frequency response over the whole structure. However, the local frequency response is quite important for some practical problems [21]. The frequency response at specified points or surfaces should be taken into the objective function of dynamic structural optimization to suppress the vibration on these points or surfaces. In this paper, minimizing the square of resonance amplitude at the specified point is selected as the optimization objective to achieve the aim. On the other hand, the consumption of CLD material is limited strictly to reach the light weight of the structure. The topology optimization problem is thus formulated as

$$
\begin{array}{ll}
\min : & s=\sum_{i=1}^{m} w_{i} X^{2}\left(\omega_{i}\right) \\
\text { s.t: } & V_{0}-\sum_{e=1}^{n} p_{e} V_{e}=0 \\
& p_{e}=\{0,1\}, \quad e=1,2, \ldots, n,
\end{array}
$$

where $p_{e}$ is the design variables, and it can be assigned 1 or 0 . $p_{e}=1$ denotes that the eth element is bonded with CLD material, while $p_{e}=0$ denotes that $e$ th element is not bonded with CLD material. $n$ is the number of elements and $m$ is the number of points. $w_{i}$ is the $i$ th weighting coefficient. $s$ is the objective function. $V_{e}$ is the volume of an individual element and $V_{0}$ is the volume constraint for the consumption of CLD material.

\section{The Sensitivity Analysis}

The sensitivity analysis of objective function with respect to the design variables is required to solve the previous optimization problem. From (9), it can be seen that the displacement response at a specified point depends on resonant frequency and the mode damping ratio and mode shape normalized with respect to the mass matrix. Furthermore, the sensitivity of objective function with respect to the design variables can be derived from (10):

$$
\begin{aligned}
\frac{\partial s}{\partial p_{e}}=2 \sum_{i=1}^{a} w_{i} X\left(\omega_{i}\right)( & \left(\frac{\partial \varphi_{i}}{\partial p_{e}} \varphi_{i}^{T}+\varphi_{i} \frac{\partial \varphi_{i}^{T}}{\partial p_{e}}\right) \frac{F}{2 \zeta_{i} \omega_{i}^{2}} \\
& -2 \zeta_{i} \frac{\partial \omega_{i}^{2}}{\partial p_{e}} \frac{\varphi_{i} \varphi_{i}^{T} F}{\left(2 \zeta_{i} \omega_{i}^{2}\right)^{2}} \\
& \left.-2 \omega_{i}^{2} \frac{\partial \zeta_{i}}{\partial p_{e}} \frac{\varphi_{i} \varphi_{i}^{T} F}{\left(2 \zeta_{i} \omega_{i}^{2}\right)^{2}}\right)
\end{aligned}
$$

In (11), the first term represents the partial derivative of eigenvector to the design variable. The second term represents the partial derivative of eigenvalue to the design variable. The third term denotes the partial derivative of modal damping ratio to the design variable. In the conventional sensitivity analysis, the modal damping ratio is often 
assumed as a constant [16]. Therefore, the third term is not considered in

$$
\begin{aligned}
\frac{\partial s}{\partial p_{e}}=2 \sum_{i=1}^{a} w_{i} X\left(\omega_{i}\right)( & \left(\frac{\partial \varphi_{i}}{\partial p_{e}} \varphi_{i}^{T}+\varphi_{i} \frac{\partial \varphi_{i}^{T}}{\partial p_{e}}\right) \frac{F}{2 \zeta_{i} \omega_{i}^{2}} \\
& \left.-2 \zeta_{i} \frac{\partial \omega_{i}^{2}}{\partial p_{e}} \frac{\varphi_{i} \varphi_{i}^{T} F}{\left(2 \zeta_{i} \omega_{i}^{2}\right)^{2}}\right) .
\end{aligned}
$$

Equation (12) is the conventional sensitivity analysis. In fact, the modal damping ratio in CLD system always varies in optimization process due to the layout modification of CLD treatment on structural surface. In view of that, the improved sensitivity analysis method is developed and the modal damping ratio is not a constant in iteration process any more. Equation (11) is used to calculate the sensitivity for each element accurately.

So far, some efficient methods have been used to calculate partial derivative of eigenvector, such as the finite difference method, the modal method, the modal truncation method, Nelson's method, and an improved modal method. In this paper, the modal truncation method is used to calculate partial derivative of eigenvector. According to the modal truncation method, the derivative of eigenvector is written as

$$
\frac{\partial \varphi_{i}}{\partial p_{e}}=\sum_{k=1}^{m} \alpha_{i k} \varphi_{k},
$$

where

$$
\begin{gathered}
\alpha_{i i}=-\frac{1}{2} \varphi_{i}^{T} \frac{\partial \mathbf{M}}{\partial p_{e}} \varphi_{i}=-\frac{1}{2} \varphi_{i}^{T}\left(\mathbf{M}_{v}^{e}+\mathbf{M}_{c}^{e}\right) \varphi_{i}, \\
\alpha_{i k}=\frac{\varphi_{k}^{T}\left(\partial \mathbf{K} / \partial p_{e}-\omega_{i}^{2}\left(\partial \mathbf{M} / \partial p_{e}\right)\right) \varphi_{i}}{\omega_{i}^{2}-\omega_{k}^{2}} \\
=\frac{\varphi_{k}^{T}\left(\left(\mathbf{K}_{v}^{e}+\mathbf{K}_{c}^{e}\right)-\omega_{i}^{2}\left(\mathbf{M}_{v}^{e}+\mathbf{M}_{c}^{e}\right)\right) \varphi_{i}}{\omega_{i}^{2}-\omega_{k}^{2}}, \quad i \neq k .
\end{gathered}
$$

The eigenvalue sensitivity is

$$
\begin{aligned}
\frac{\partial \omega_{i}^{2}}{\partial p_{e}} & =\varphi_{i}^{T}\left(\frac{\partial \mathbf{K}}{\partial p_{e}}-\omega_{i}^{2} \frac{\partial \mathbf{M}}{\partial p_{e}}\right) \varphi_{i} \\
& =\varphi_{i}^{T}\left(\left(\mathbf{K}_{v}^{e}+\mathbf{K}_{c}^{e}\right)-\omega_{i}^{2}\left(\mathbf{M}_{v}^{e}+\mathbf{M}_{c}^{e}\right)\right) \varphi_{i} .
\end{aligned}
$$

The derivative of the $i$ th modal loss factor can be obtained from (3):

$$
\frac{\partial \eta_{i}}{\partial p_{e}}=\eta_{v} \frac{E_{s v}^{i e} E_{s}^{i}-\left(E_{s v}^{i e}+E_{s c}^{i e}\right) E_{s v}^{i}}{\left(E_{s}^{i}\right)^{2}} .
$$

Thus, the derivative of the $i$ th modal damping ratio is expressed by applying (5):

$$
\frac{\partial \zeta_{i}}{\partial p_{e}}=\frac{\eta_{v}}{2} \frac{E_{s v}^{i e} E_{s}^{i}-\left(E_{s v}^{i e}+E_{s c}^{i e}\right) E_{s v}^{i}}{\left(E_{s}^{i}\right)^{2}} .
$$

In order to eliminate checkerboard patterns in layout optimization, the mesh-independency filter [26] is used to modify the element sensitivities.

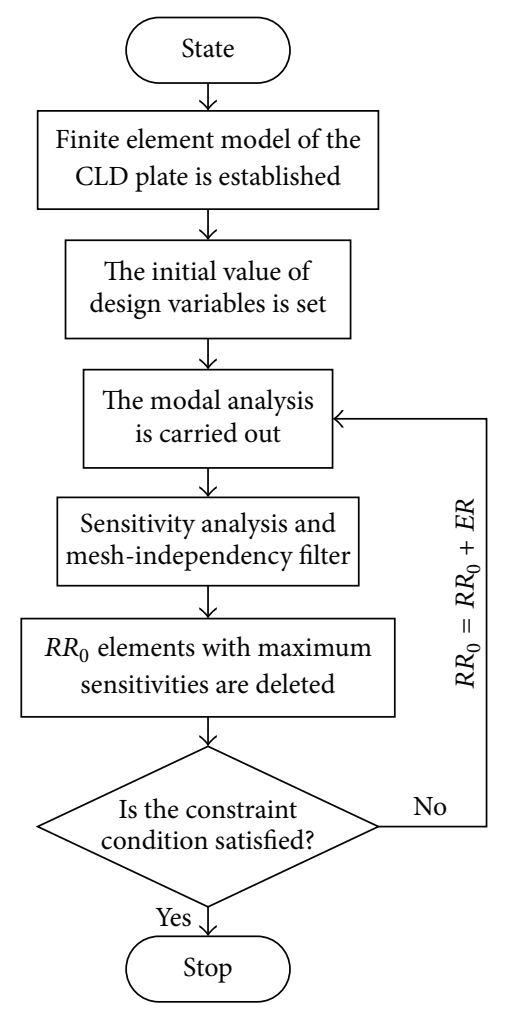

FIGURE 2: Flowchart of the topology optimization.

\section{Optimization Strategy}

The evolutionary structural optimization (ESO) is used to solve the proposed optimization problem. The ESO method has been widely used in structural optimization since Xie and Steven [27] presented this method. The advantages of ESO method lie in its simplicity in achieving shape and topology optimization for both static and dynamic problems. The flowchart for implementation of the topology optimization procedures is shown in Figure 2. The evolutionary procedure for topology optimization of CLD structures to minimize the square of displacement response at resonant frequency for the specified point is summarized as follows.

(1) The finite element model of the plate with CLD treatment is established.

(2) The initial value of design variables is set. The constraint consumption of CLD material $V^{*}$, the initial removed elements of CLD material $R R_{0}$, and evolutionary ratio ER are determined, respectively.

(3) The modal analysis of the plate with CLD treatment is completed.

(4) The sensitivities of objective function to design variable are calculated and the mesh-independency filter method is used to modify the sensitivity of the element.

(5) Delete $R R_{0}$ elements with minimum sensitivity values. 
TABLE 1: Physical and geometrical parameters of CLD plate.

\begin{tabular}{|c|c|c|c|c|c|c|}
\hline & $\begin{array}{l}\text { Length } \\
(\mathrm{m})\end{array}$ & $\begin{array}{l}\text { Width } \\
(\mathrm{m})\end{array}$ & $\begin{array}{c}\text { Thickness } \\
(\mathrm{mm})\end{array}$ & $\begin{array}{l}\text { Young's modulus } \\
(\mathrm{MPa})\end{array}$ & $\begin{array}{l}\text { Density } \\
\left(\mathrm{kg} / \mathrm{m}^{3}\right)\end{array}$ & Poisson's ratio \\
\hline Base layer & 0.2 & 0.1 & 0.8 & 70 & 2800 & 0.3 \\
\hline Damping layer & 0.2 & 0.1 & 0.05 & 12 & 1200 & 0.495 \\
\hline Constrained layer & 0.2 & 0.1 & 0.13 & 70 & 2700 & 0.3 \\
\hline
\end{tabular}

TABLE 2: A comparison between CLD system with full coverage and CLD system with optimal layout of CLD material on plate.

\begin{tabular}{lccc}
\hline & Full coverage & The conventional sensitivity analysis & The improved sensitivity analysis \\
\hline Frequency $(\mathrm{Hz})$ & 19.80 & 20.99 & 19.02 \\
Modal damp ratio & $1.53 \times 10^{-3}$ & $3.18 \times 10^{-3}$ & $7.16 \times 10^{-3}$ \\
Objective function $\left(\mathrm{m}^{2}\right)$ & $3.40 \times 10^{-3}$ & $9.05 \times 10^{-4}$ & $2.18 \times 10^{-4}$ \\
\hline
\end{tabular}

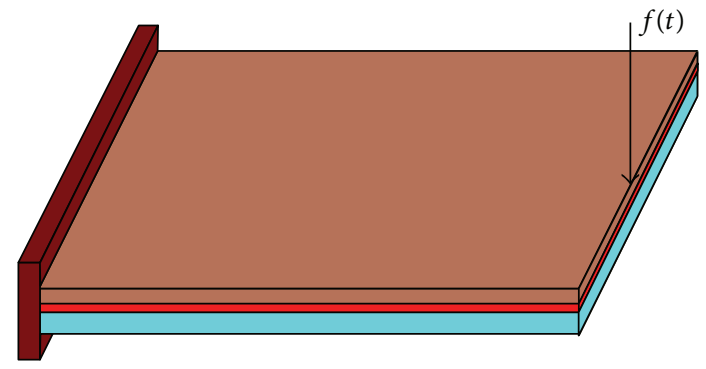

FIGURE 3: The cantilever CLD plate.

(6) Check the constraint condition; if the constraint condition is satisfied, then stop calculation; or update $R R_{0}$ by the formula of $R R_{0}=R R_{0}+\mathrm{ER}$.

(7) Repeat procedures (3)-(7) until the constraint condition is satisfied.

\section{Numerical Examples}

5.1. The Cantilever Plate/CLD System. Figure 3 is a cantilever plate/CLD system. The main physical and geometrical parameters of the base plate, the damping layer, and constrained layer are listed in Table 1 . The loss factor of viscoelastic material is 0.5. A unit harmonic force is applied at the midpoint of the free edge. The range of excitation frequency is $[10 \mathrm{~Hz}-40 \mathrm{~Hz}]$, which includes the first-order resonant frequency $(19.80 \mathrm{~Hz})$. The design objective is considered as the square of the first-order resonant amplitude at the loading point.

The volume consumption of CLD material is limited to $50 \%$ of full coverage in optimization process. The objective function is to minimize the square of displacement response at first-order resonant frequency for the specified loading point. In order to obtain the symmetric results, only the sensitivity for the upper half of the cantilever CLD plate is calculated and the sensitivity for the whole cantilever CLD plate is obtained through the symmetry constraint. The ESO method is used to solve the proposed optimization problem. According to ESO method, when iteration number increases, the rejection ratio also increases.
Figures 4 and 5 demonstrate the topology evolving processes by using conventional sensitivity analysis and the improved sensitivity analysis, respectively. It is seen that some holes appear in the optimal layout of CLD material when the improved sensitivity analysis is used and the boundary line of CLD material is not smooth anymore. Figures 6, 7, and 8 show iteration histories of the first-mode frequency, modal damping ratio, and the objective function, respectively. It is noted that the modal damping ratio in CLD system by using improved sensitivity analysis increases more rapidly than by using conventional sensitivity analysis. Meanwhile, the objective function decreases rapidly to a lower value than that obtained from the conventional sensitivity analysis. Edge effect should be considered as the main reason to explain the phenomenon. The shear deformation occurring in the damping layer is responsible for the dissipation of energy and cutting both the damping layer and constrained layer will increase the shear deformation at relative position [13]. This implies that the improved sensitivity analysis is more accurate on the layout optimization of CLD material on the cantilever plate, which also validates the effectiveness of the proposed improved sensitivity analysis.

Table 2 compares information before and after optimization. Data demonstrates that the first-mode frequency decreases by $0.3 \%$, the modal damping ratio with optimal layout is 4.67 times that with full coverage, and the objective function is only $0.06 \%$ of CLD system with full coverage. It is noticed that, in optimization process, only 50\% CLD material is applied on the surface of the cantilever plate. This means that dynamic response in first mode can be suppressed significantly with an optimal layout of CLD material and much less extra weight. It is usually required in engineering applications due to structural light weight trend.

5.2. The Clamped Plate/CLD System. Figure 9 shows a clamped plate/CLD system with all clamped plates on four sides. The base plate is made of steel, with the length of $0.6 \mathrm{~m}$, width of $0.4 \mathrm{~m}$, and thickness of $0.0015 \mathrm{~m}$. Young's modulus, density, and Poisson's ratio are $212 \mathrm{GPa}, 7860 \mathrm{~kg} / \mathrm{m}^{3}$, and 0.3 , respectively. The same CLD material as the previous example is applied on the plate. A unit harmonic force is applied at the center of the plate. The range of the exciting frequency 


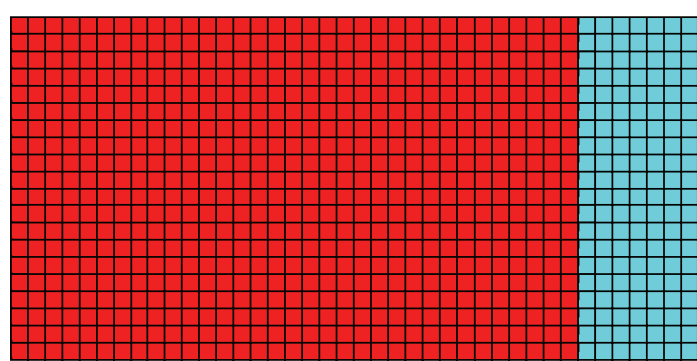

(a)

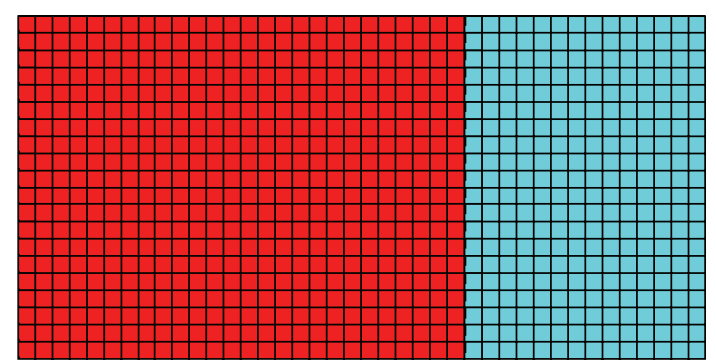

(b)

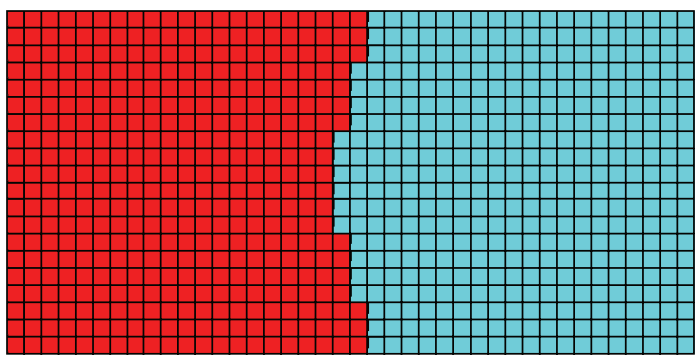

(c)

FIGURE 4: Topology evolving process using conventional sensitivity analysis. (a) Iteration 7 (rejection ratio 17.5\%). (b) Iteration 14 (rejection ratio 35\%). (c) Iteration 20 (rejection ratio 50\%).

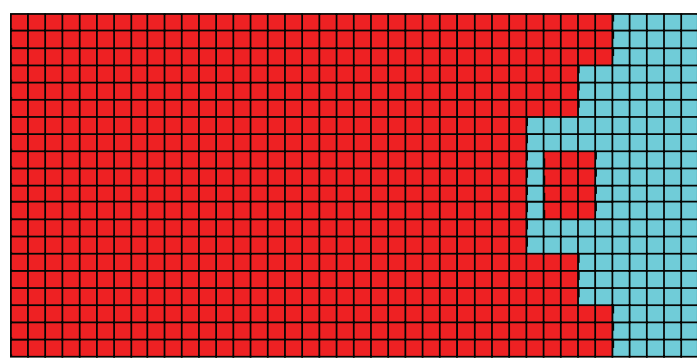

(a)

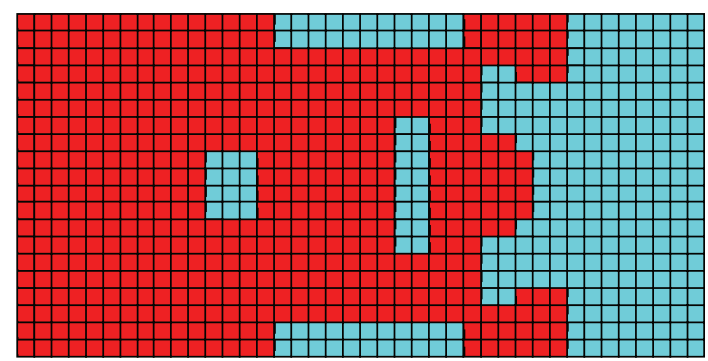

(b)

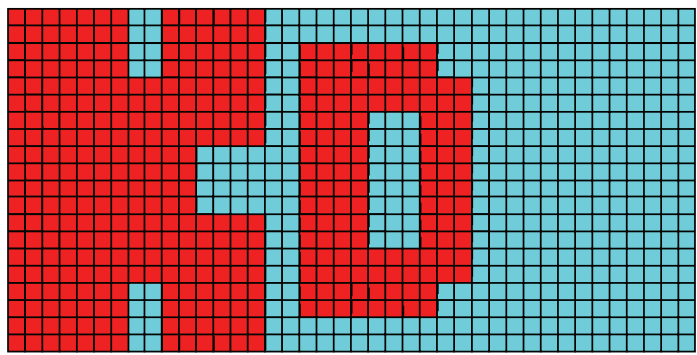

(c)

Figure 5: Topology evolving process using improved sensitivity analysis. (a) Iteration 7 (rejection ratio 17.5\%). (b) Iteration 14 (rejection ratio $35 \%)$. (c) Iteration 20 (rejection ratio 50\%).

is selected from $50 \mathrm{~Hz}$ to $150 \mathrm{~Hz}$, which includes the firstand second-mode frequency. The objective function is the sum of the amplitude squares for the first-order and secondorder displacement response at the loading point. Setting the weighted factors for both the first and second mode as 0.5 , the objective function can be calculated by $w_{1} X^{2}\left(\omega_{1}\right)+$ $w_{2} X^{2}\left(\omega_{2}\right), w_{1}=w_{2}=0.5$. In this example, only the sensitivity for the upper left quadrant of the clamped CLD plate is calculated and the sensitivity for the whole clamped CLD plate is obtained through the symmetry constraint. The volume consumption of CLD material is limited to $50 \%$ of full coverage in optimization process. According to ESO method, when iteration number increases, the rejection ratio also increases.

The topology evolving processes by conventional sensitivity analysis and improved sensitivity analysis are diagrammed 
TABLE 3: A comparison between CLD system with full coverage and the layout of CLD material.

\begin{tabular}{lccc}
\hline & Full coverage & The conventional sensitivity analysis & The improved sensitivity analysis \\
\hline Weighted frequency $(\mathrm{Hz})$ & 83.44 & 82.11 & 81.50 \\
Weighted modal damp ratio & $2.4 \times 10^{-3}$ & $2.57 \times 10^{-3}$ & $3.91 \times 10^{-3}$ \\
Objective function $\left(\mathrm{m}^{2}\right)$ & $3.84 \times 10^{-7}$ & $3.35 \times 10^{-7}$ & $1.79 \times 10^{-7}$ \\
\hline
\end{tabular}

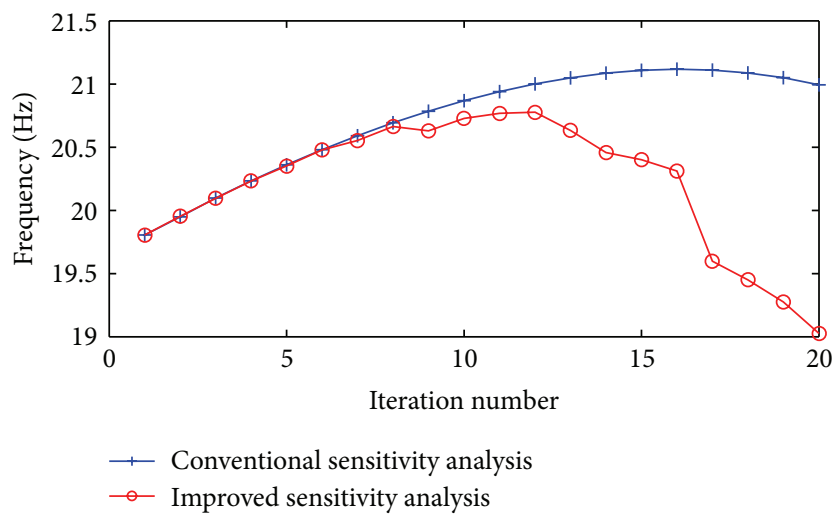

FIGURE 6: Iteration histories of the first-order frequency.

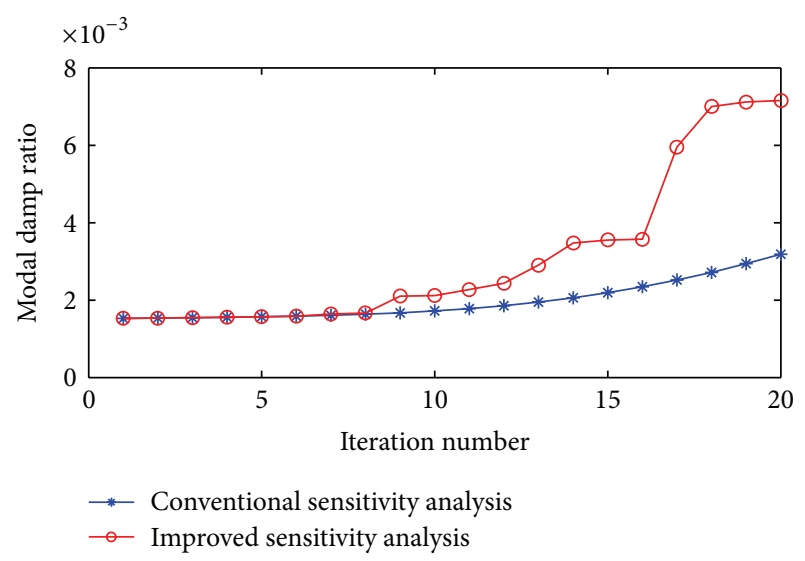

FIGURE 7: Iteration histories of modal damp ratio.

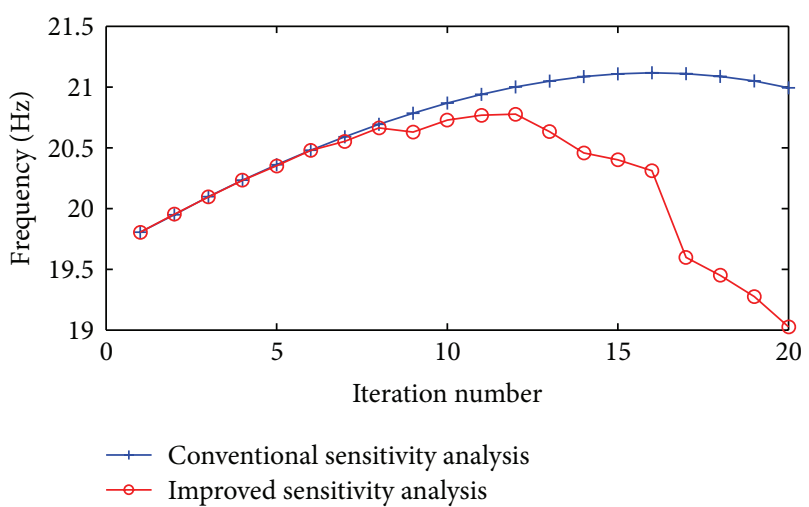

FIGURE 8: Iteration histories of objective function value.

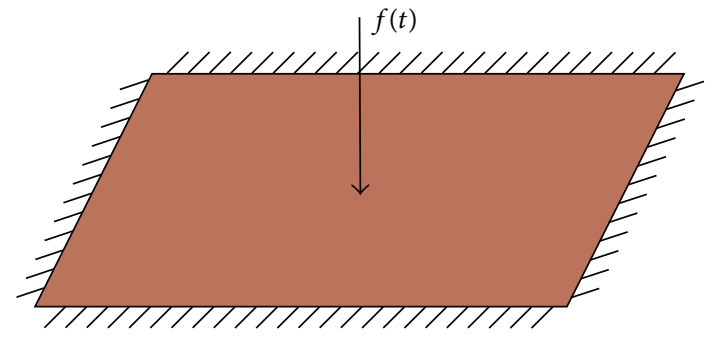

FIgURE 9: The clamped plate/CLD system.

in Figures 10 and 11, respectively. Figure 12 demonstrates the iteration histories of the weighted frequency $\left(w_{1} \omega_{1}+w_{2} \omega_{2}\right)$. Figure 13 shows the iteration histories of the weighted modal damping ratio $\left(w_{1} \zeta_{1}+w_{2} \zeta_{2}\right)$. Figure 14 shows the iteration histories of the objective function. It is obvious that the weighted frequency by using the improved sensitivity analysis decreases much more than that using the conventional sensitivity analysis. It should be corresponding with actual situation. Additionally, the weighted modal damping ratio of CLD system increases rapidly and reaches a higher value than that using the conventional sensitivity analysis. In the same way, the objective function decreases to a lower value than that using the conventional sensitivity analysis. Edge effect is also the main reason to explain the phenomenon. Some holes appear in the optimal layout of CLD material using the improved sensitivity analysis and they increase the shear deformation at those positions. Therefore, the layout of CLD material obtained by improved sensitivity analysis is more accurate in details than that obtained by conventional sensitivity analysis. The influence of the improved sensitivity analysis on the layout of CLD material and vibration suppression in plates is significant.

Table 3 shows that the improved sensitivity analysis results in obvious increase in the modal damping ratio of the clamped plate with CLD treatment and a rapid drop in the displacement response for the first and the second mode. It is seen that the weighted modal damping ratio using improved sensitivity analysis increases 52\% more than that using conventional sensitivity analysis. Of course, whether the conventional or improved sensitivity analysis is used in topology optimization process, it is obvious that the optimal layout of CLD material on the plate may result in the effective vibration suppression at a cost of $50 \%$ CLD material applied. 


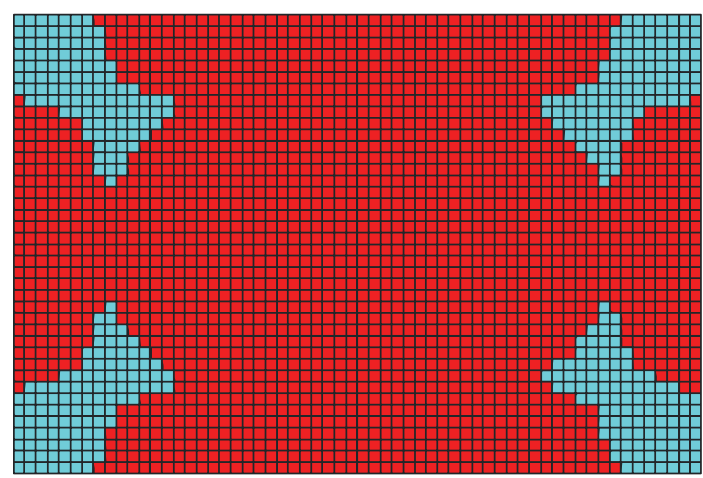

(a)

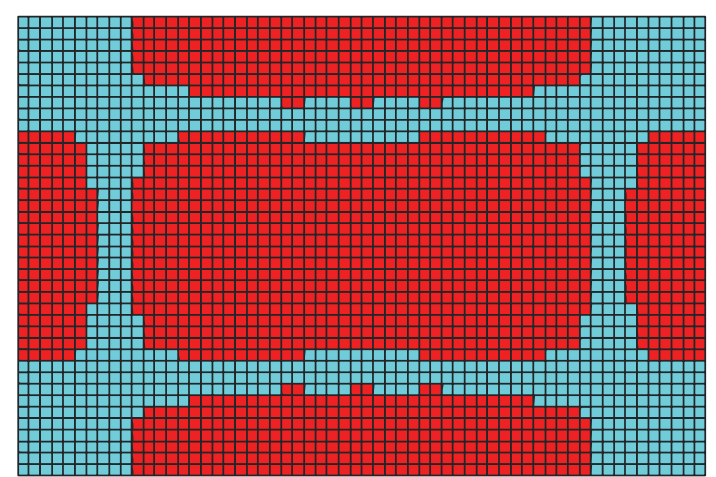

(b)

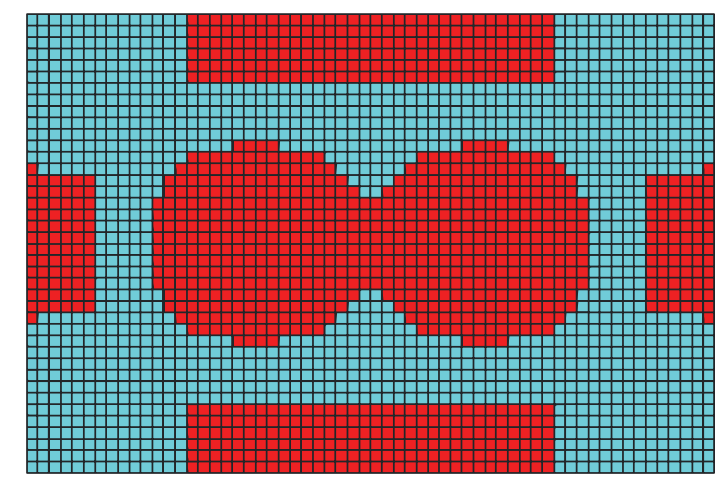

(c)

FIGURE 10: Topology evolving process using conventional sensitivity analysis. (a) Iteration 14 (rejection ratio 17.5\%). (b) Iteration 28 (rejection ratio 35\%). (c) Iteration 40 (rejection ratio 50\%).

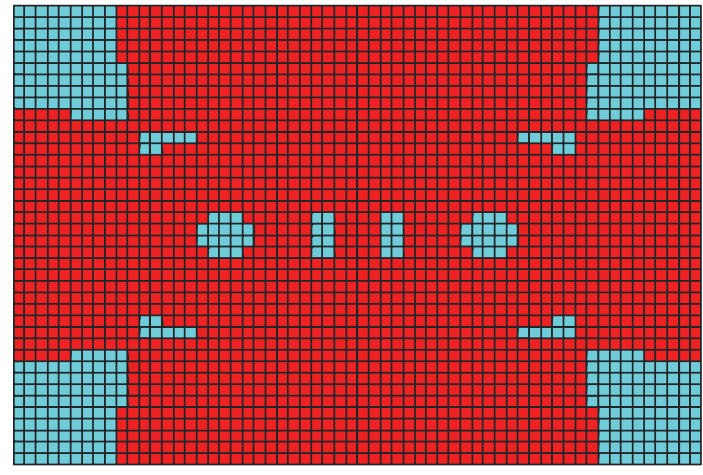

(a)

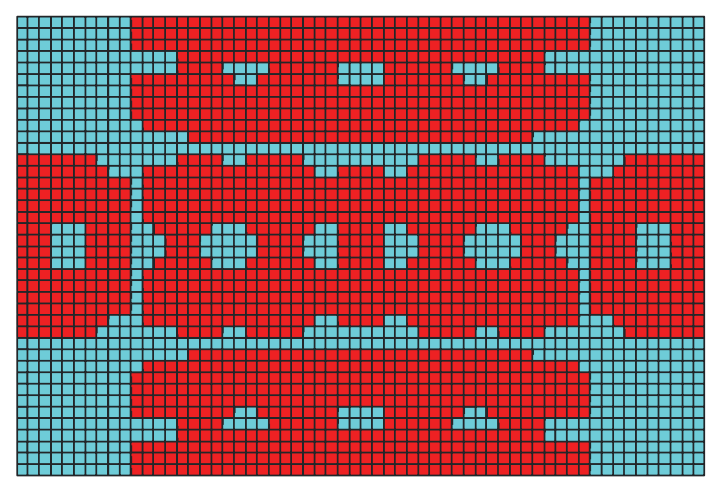

(b)

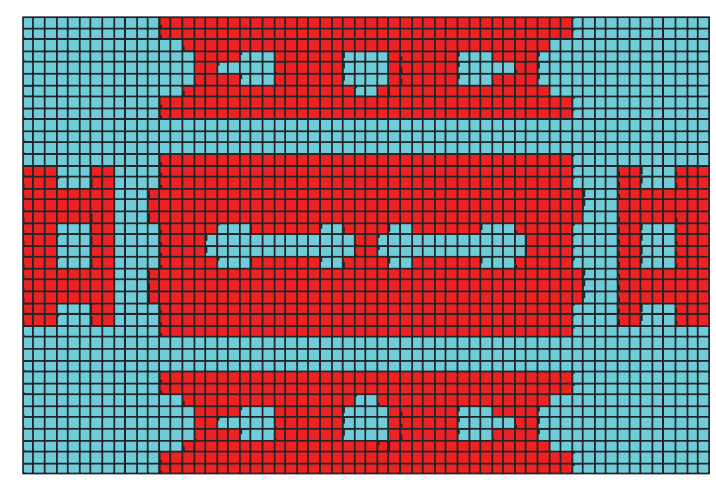

(c)

FIGURE 11: Topology evolving process using improved sensitivity analysis. (a) Iteration 14 (rejection ratio 17.5\%). (b) Iteration 28 (rejection ratio $35 \%$ ). (c) Iteration 40 (rejection ratio 50\%). 


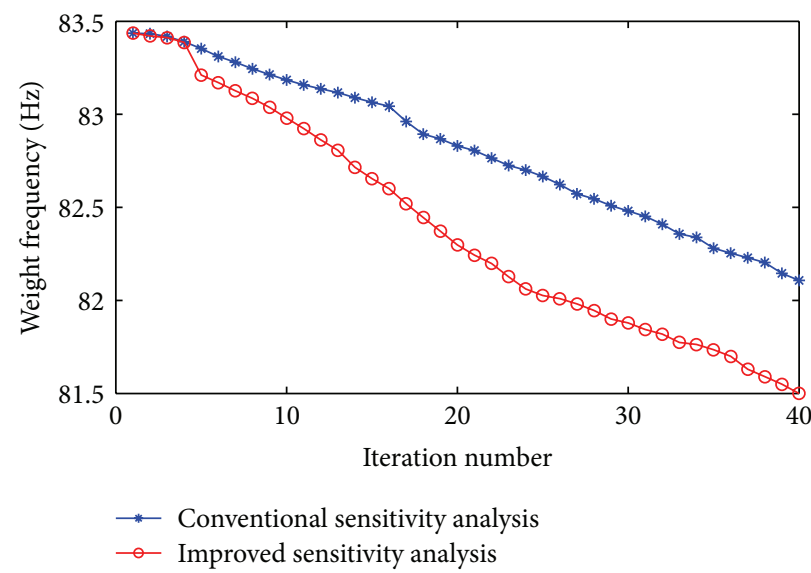

FIGURE 12: Iteration histories of the frequency.

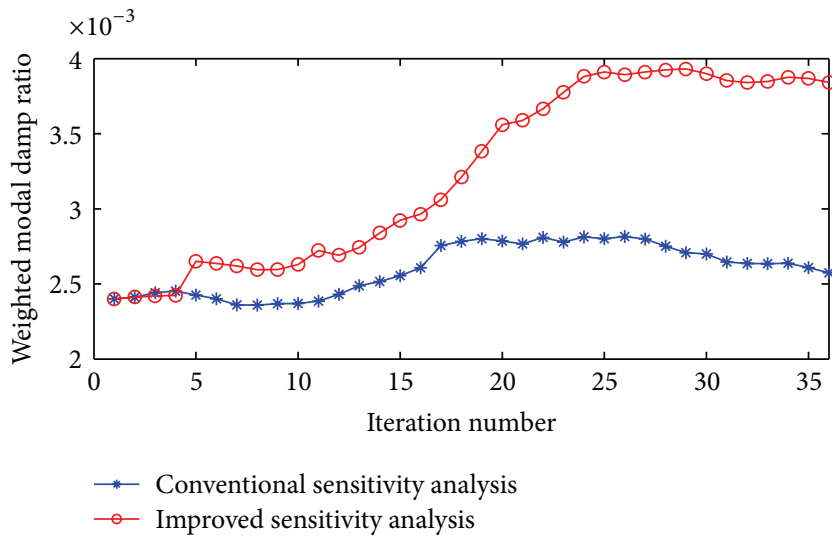

FIGURE 13: Iteration histories of the modal damp ratio.

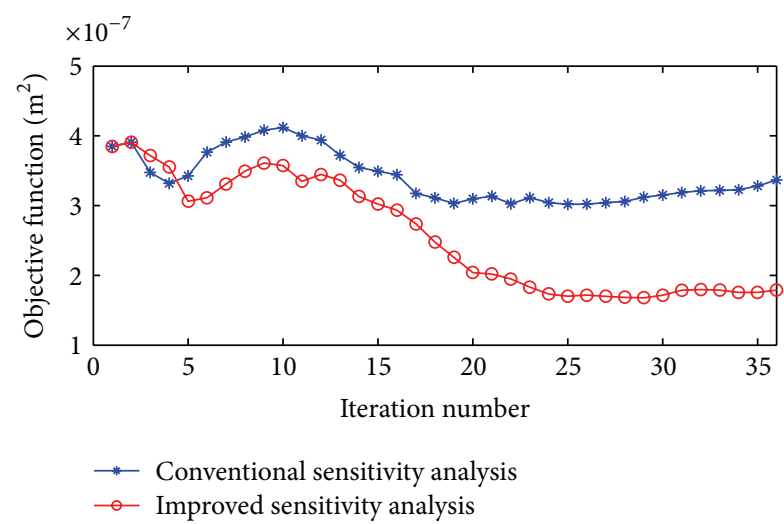

FIgURE 14: Iteration histories of the modal damp ratio.

\section{Experimental Verification}

The three samples are prepared and the vibration responses are measured in order to verify the effectiveness of the optimal layout of CLD material for the first example shown in Figure 3. These measured samples are shown in Figure 15.
Figure 15(a) is the cantilever plate with full coverage. Figure 15(b) is the optimal layout by using conventional sensitivity analysis. Figure 15 (c) is the optimal layout by using improved sensitivity analysis. Three holes are drilled to clamp the sample.

In experimental process, a hammer "PCB $086 \mathrm{C} 03$ " is used to supply an excitation for these cantilever plates with CLD treatment. The displacement response is measured by using a laser displacement sensor "KEYENCE LK- H080" and all dates are recorded and analyzed by LMS test system. The excitation point and the measure point are at the same place, which locate at the middle of the free end of cantilever plate. The experimental setup is shown in Figure 16.

The frequency response comparison between numerical and experimental results of the cantilever plates is shown in Figure 17. The results obtained indicate that the experimental results are in agreement with numerical calculations. On the other hand, it is noted that the optimal layout of CLD material on the cantilever plate can significantly reduce the displacement response at the peak of the first natural frequency. Meanwhile, the consumption of CLD material is only $50 \%$ of full coverage CLD material. In addition, it is seen that the optimal layout of CLD treatment on the cantilever plate using the improved sensitivity analysis can indeed result in more effective vibration suppression than that using the conventional sensitivity analysis.

\section{Conclusion}

In this paper, an improved sensitivity analysis method is proposed and used to obtain the optimal layout of CLD material on plates. The optimization model is established and the square of the displacement response at the first resonant frequency or other resonant frequencies is considered as the objective function to suppress structural vibration in a small extra mass of CLD material. The ESO method is adopted to search the optimal layout of CLD material on the plates. The numerical examples are given and the experiments are carried out to verify the effectiveness of the improved sensitivity analysis. The experimental and theoretical results show that 


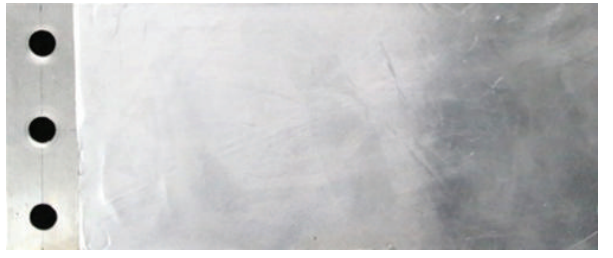

(a)

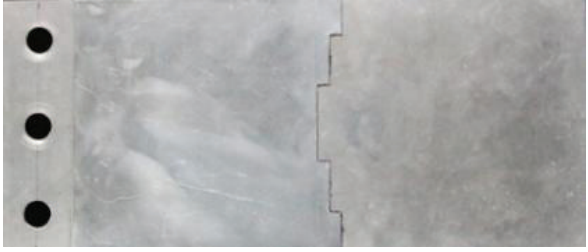

(b)

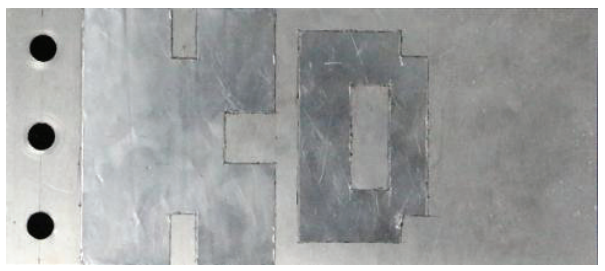

(c)

Figure 15: The experimental samples. (a) Full coverage. (b) The optimal layout by using conventional sensitivity analysis. (c) The optimal layout by using improved sensitivity analysis.

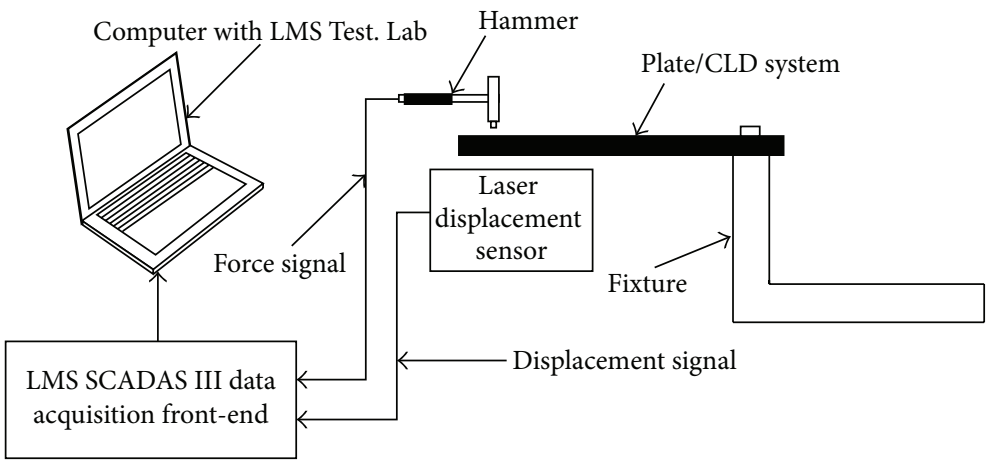

(a)

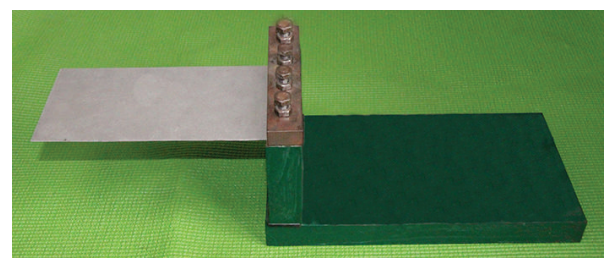

(b)

FIgURE 16: The experimental setup. (a) Schematic drawing. (b) The picture of the plate clamped in its fixture.
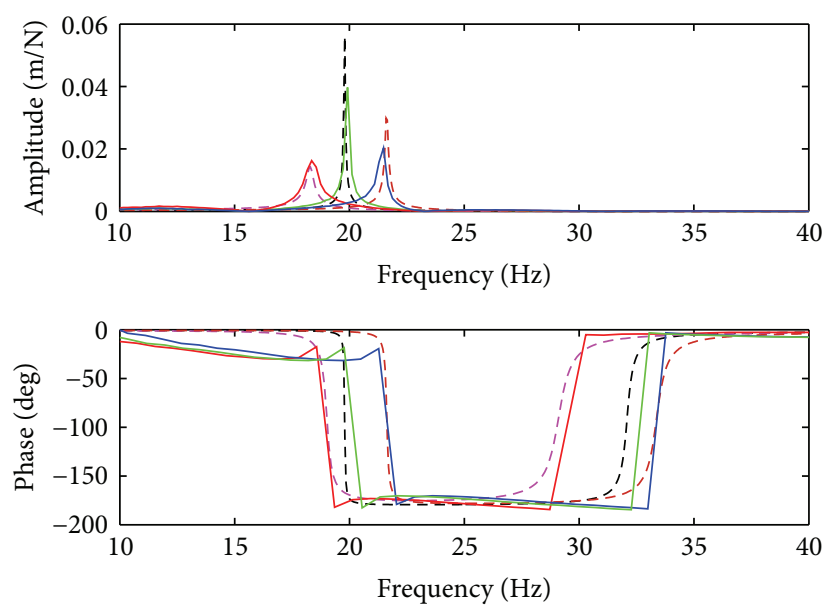

- - - Full coverage-numerical
- - - Improved sensitivity analysis-numerical
- - - Conventional sensitivity analysis-numerical
- Full coverage-experimental
— Improved sensitivity analysis-experimental
— Conventional sensitivity analysis-experimenta

FIGURE 17: Frequency response comparison between numerical and experimental results of the cantilever plates. 
the optimal layout of CLD treatment on plates can significantly reduce the displacement response for one or more resonant frequencies in a frequency range by the proposed topology optimization method. Moreover, by contrast, the improved sensitivity analysis proposed in this paper can increase the modal damping ratio of CLD system and supply more accurate layout of CLD treatment. This results in more effective vibration suppression in plates with CLD treatment.

\section{Conflict of Interests}

The authors declare that there is no conflict of interests regarding the publication of this paper.

\section{Acknowledgments}

This work was supported by the Natural Science Foundation of China (Grant no. 50775225) and the State Key Laboratory of Mechanical Transmission, Chongqing University (Grant no. 0301002109165). These financial supports are gratefully acknowledged.

\section{References}

[1] E. M. Kerwin, "Damping of flexural waves by a constrained viscoelastic layer," The Journal of the Acoustical Society of America, vol. 31, no. 7, pp. 952-962, 1959.

[2] R. A. DiTaranto and W. Blasingame, "Composite loss factors of selected laminated beams," Journal of the Acoustical Society of America, vol. 40, pp. 187-194, 1965.

[3] D. J. Mead and S. Markus, "The forced vibration of a three-layer, damped sandwich beam with arbitrary boundary conditions," Journal of Sound and Vibration, vol. 10, no. 2, pp. 163-175, 1969.

[4] D. S. Nokes and F. C. Nelson, "Constrained layer damping with partial coverage," Shock and Vibration, vol. 38, pp. 5-10, 1968.

[5] A. K. Lall, N. T. Asnani, and B. C. Nakra, "Vibration and damping analysis of rectangular plate with partially covered constrained viscoelastic layer," Journal of Vibration, Acoustics, Stress, and Reliability in Design, vol. 109, no. 3, pp. 241-247, 1987.

[6] A. K. Lall, N. T. Asnani, and B. C. Nakra, "Damping analysis of partially covered sandwich beams," Journal of Sound and Vibration, vol. 123, no. 2, pp. 247-259, 1988.

[7] S. Kodiyalam and J. Molnar, "Optimization of constrained viscoelastic damping treatments for passive vibration control," in Proceedings of the 33rd AIAA/ASME/ASCE/AHS/ASC Structures, Structural Dynamics and Materials Conference, pp. 1479-1487, 1993.

[8] A. Lumsdaine, "Topology optimization of constrained damping layer treatments," in Proceedings of the ASME International Mechanical Engineering Congress and Exposition, pp. 149-156, New Orleans, La, USA, November 2002.

[9] H. Zheng, C. Cai, G. S. H. Pau, and G. R. Liu, "Minimizing vibration response of cylindrical shells through layout optimization of passive constrained layer damping treatments," Journal of Sound and Vibration, vol. 279, no. 3-5, pp. 739-756, 2005.

[10] R. A. S. Moreira and J. D. Rodrigues, "Partial constrained viscoelastic damping treatment of structures: a modal strain energy approach," International Journal of Structural Stability and Dynamics, vol. 6, no. 3, pp. 397-411, 2006.
[11] Z. Li and X. Liang, "Vibro-acoustic analysis and optimization of damping structure with response surface method," Materials \& Design, vol. 28, no. 7, pp. 1999-2007, 2007.

[12] Z. Ling, X. Ronglu, W. Yi, and A. El-Sabbagh, "Topology optimization of constrained layer damping on plates using Method of Moving Asymptote (MMA) approach," Shock and Vibration, vol. 18, no. 1-2, pp. 221-244, 2011.

[13] G. Lepoittevin and G. Kress, "Optimization of segmented constrained layer damping with mathematical programming using strain energy analysis and modal data," Materials \& Design, vol. 31, no. 1, pp. 14-24, 2010.

[14] M. Ansari, A. Khajepour, and E. Esmailzadeh, "Application of level set method to optimal vibration control of plate structures," Journal of Sound and Vibration, vol. 332, no. 4, pp. 687700, 2013.

[15] S. Y. Kim, C. K. Mechefske, and I. Y. Kim, "Optimal damping layout in a shell structure using topology optimization," Journal of Sound and Vibration, vol. 332, no. 12, pp. 2873-2883, 2013.

[16] J. H. Rong, Y. M. Xie, X. Y. Yang, and Q. Q. Liang, “Topology optimization of structures under dynamic response constraints," Journal of Sound and Vibration, vol. 234, no. 2, pp. 177189, 2000.

[17] H. Zheng, C. Cai, G. S. H. Pau, and G. R. Liu, "Minimizing vibration response of cylindrical shells through layout optimization of passive constrained layer damping treatments," Journal of Sound and Vibration, vol. 279, no. 3-5, pp. 739-756, 2005.

[18] J. Pan and D. Y. Wang, "Topology optimization of truss structure with fundamental frequency and frequency domain dynamic response constraints," Acta Mechanica Solida Sinica, vol. 19, no. 3, pp. 231-240, 2006.

[19] M. Alvelid, "Optimal position and shape of applied damping material," Journal of Sound and Vibration, vol. 310, no. 4-5, pp. 947-965, 2008.

[20] G. H. Yoon, "Structural topology optimization for frequency response problem using model reduction schemes," Computer Methods in Applied Mechanics and Engineering, vol. 199, no. 2528, pp. 1744-1763, 2010.

[21] L. Shu, M. Y. Wang, Z. Fang, Z. Ma, and P. Wei, "Level set based structural topology optimization for minimizing frequency response," Journal of Sound and Vibration, vol. 330, no. 24, pp. 5820-5834, 2011.

[22] C. D. Johnson and D. A. Kienholz, "Finite element prediction of damping in structures with constrained viscoelastic layers," AIAA Journal, vol. 20, no. 9, pp. 1284-1290, 1982.

[23] E. J. Graesser and C. R. Wong, "The relationship of traditional damping measures for materials with high damping capacity: a review," in $M^{3} D$ : Mechanics and Mechanisms of Material Damping, ASTM STP 1169, pp. 316-343, 1992.

[24] C. S. Jog, "Topology design of structures subjected to periodic loading," Journal of Sound and Vibration, vol. 253, no. 3, pp. 687709, 2002.

[25] N. Olhoff and J. Du, "On topological design optimization of structures against vibration and noise emission," in Computational Aspects of Structural Acoustics and Vibration, G. Sandberg and R. Ohayon, Eds., vol. 505 of CISM International Centre for Mechanical Sciences, pp. 217-276, Springer, Vienna, Austria, 2009.

[26] O. Sigmund and J. Petersson, "Numerical instabilities in topology optimization: a survey on procedures dealing with checkerboards, mesh-dependencies and local minima," Structural Optimization, vol. 16, no. 1, pp. 68-75, 1998.

[27] Y. M. Xie and G. P. Steven, "A simple evolutionary procedure for structural optimization," Computers \& Structures, vol. 49, no. 5, pp. 885-896, 1993. 

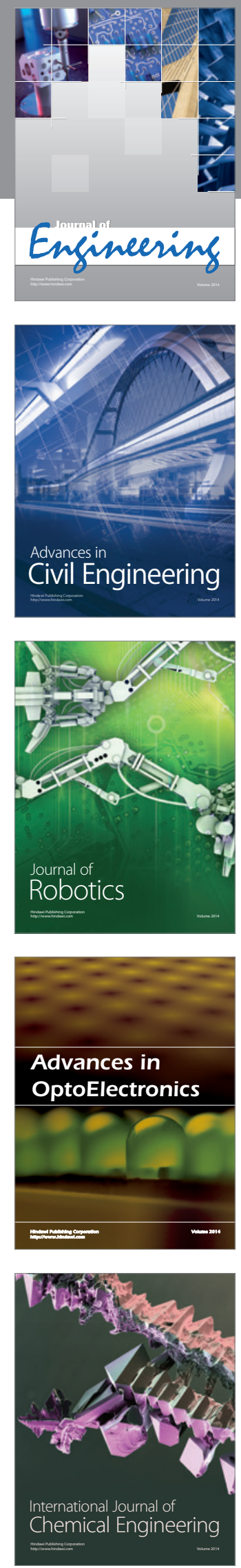

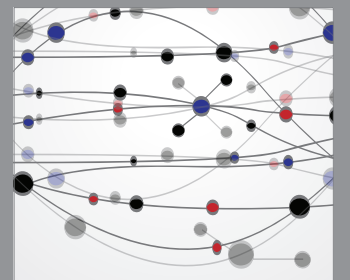

The Scientific World Journal
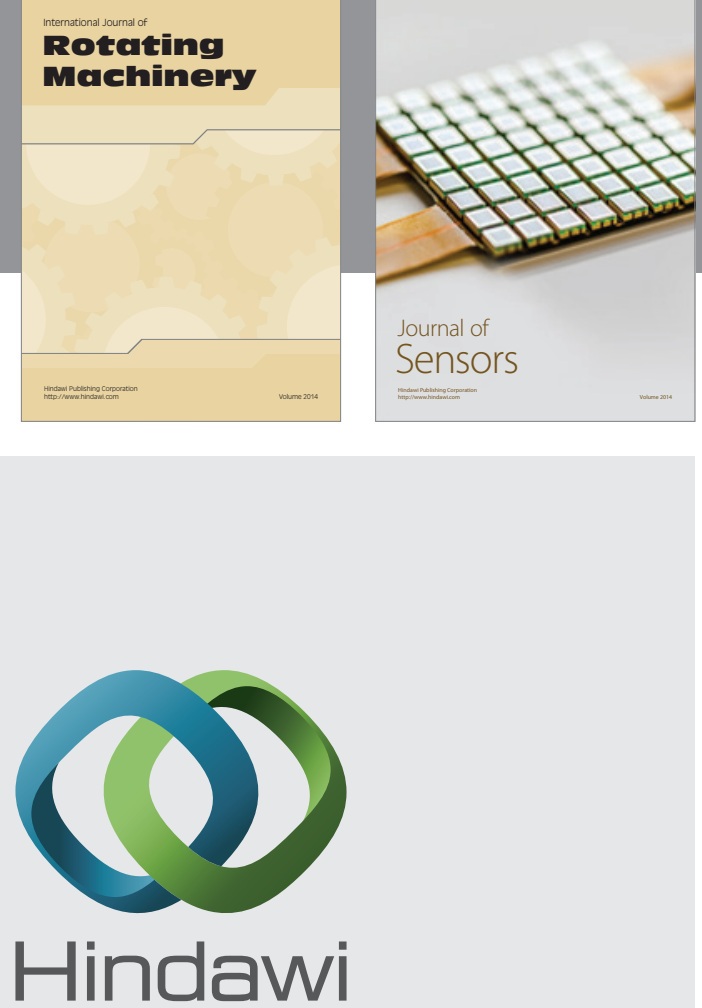

Submit your manuscripts at http://www.hindawi.com
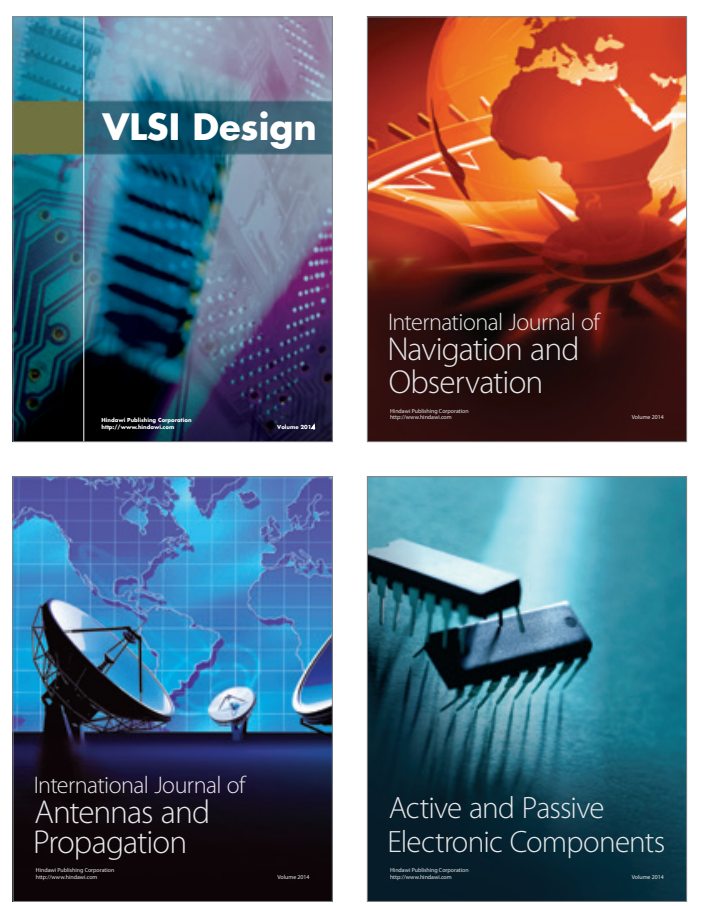
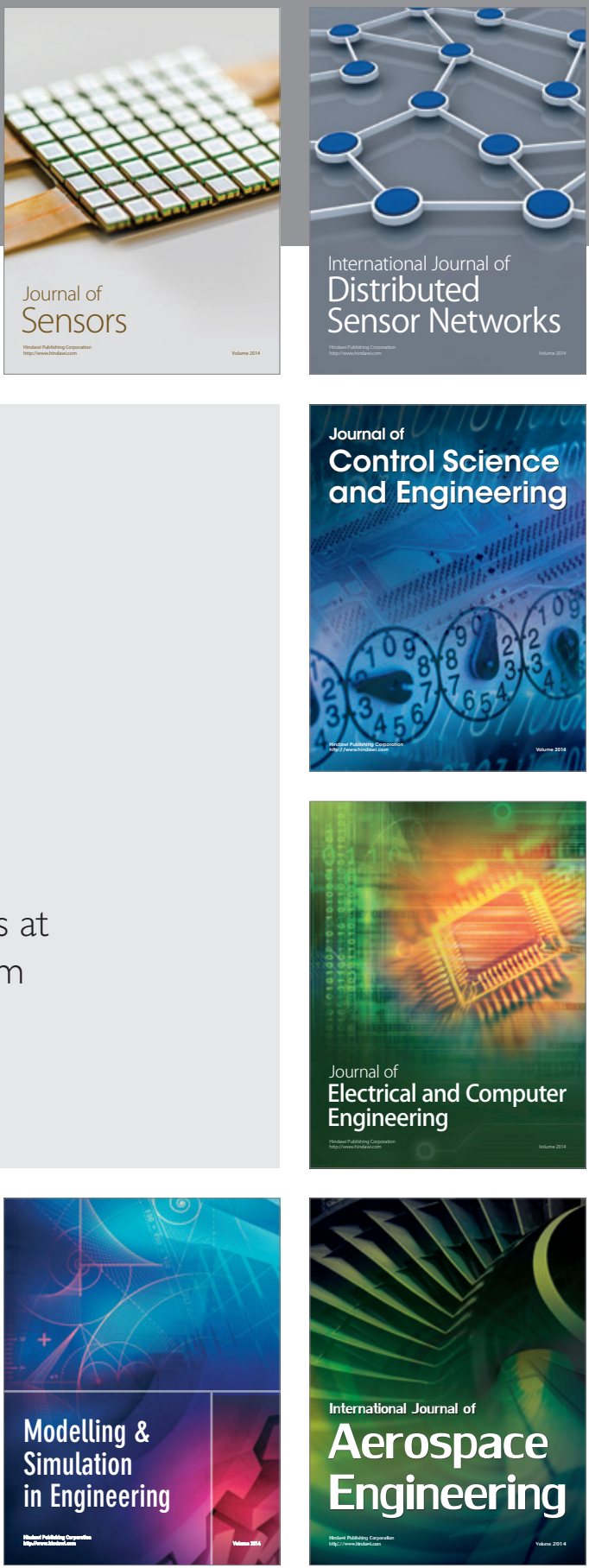

Journal of

Control Science

and Engineering
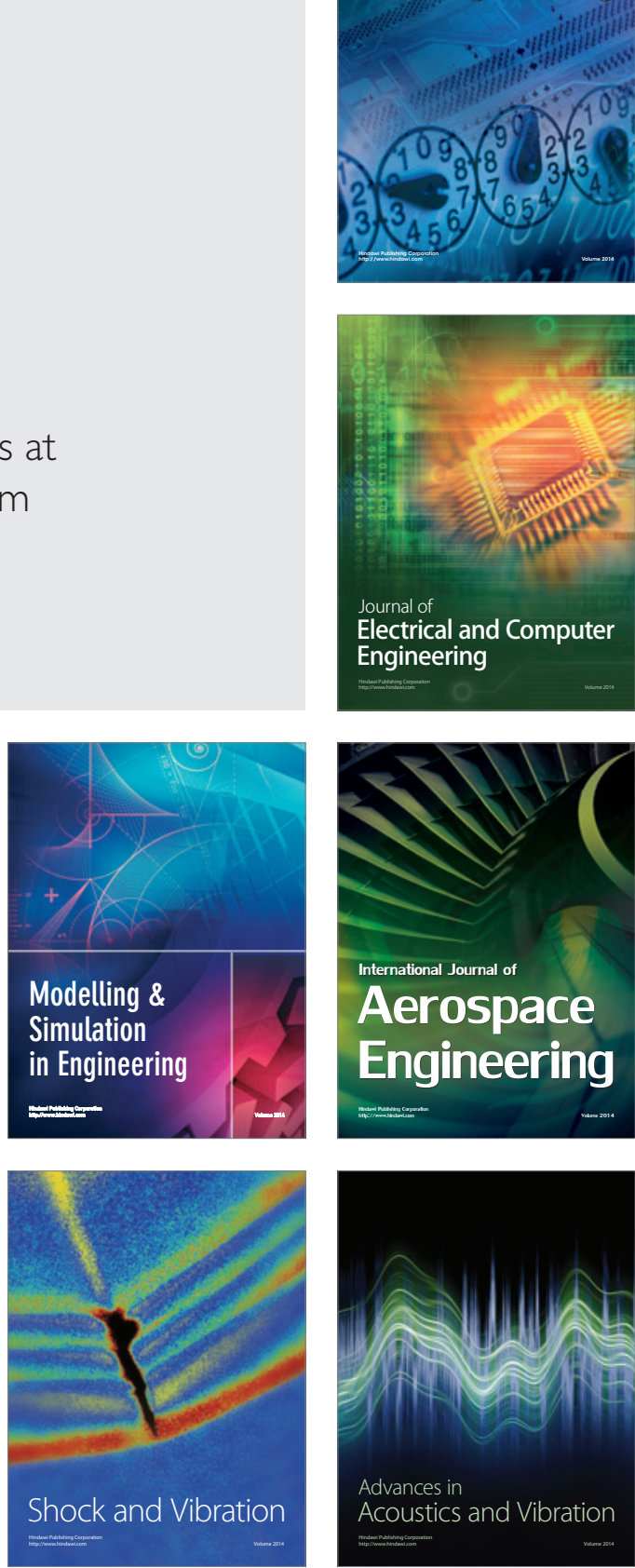